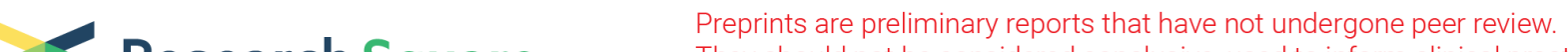 $\begin{array}{ll}\text { Research Square } & \text { They should not be considered conclusive, used to inform clinical practice, } \\ \text { or referenced by the media as validated information. }\end{array}$
}

\section{Prevalence of Erectile Dysfunction According to Chronic Comorbid Health Conditions: a Cross- sectional Study.}

\section{Giuseppe Lisco}

University of Bari Interdisciplinary Department of Medicine: Universita degli Studi di Bari Dipartimento Interdisciplinare di Medicina https://orcid.org/0000-0001-6521-8578

\section{Nicola Bartolomeo}

Università Degli Studi di Bari Dipartimento di Scienze Biomediche ed Oncologia Umana: Universita Degli

Studi di Bari Aldo Moro Dipartimento di Scienze Biomediche ed Oncologia Umana

\section{Maria Isabella Ramunni}

ASL Bari

Anna De Tullio

University of Bari Interdisciplinary Department of Medicine: Universita degli Studi di Bari Dipartimento Interdisciplinare di Medicina

\section{Matteo Domenico Carbone}

Institute of Clinical and Hormonal Research

\section{Edoardo Guastamcchia}

Università degli Studi di Bari Aldo Moro Dipartimento Interdisciplinare di Medicina: Universita degli Studi di Bari Aldo Moro Dipartimento Interdisciplinare di Medicina

\section{Vincenzo Triggiani}

Università degli Studi di Bari Aldo Moro Dipartimento Interdisciplinare di Medicina: Universita degli Studi di Bari Aldo Moro Dipartimento Interdisciplinare di Medicina

\section{Vito Angelo Giagulli ( $\nabla$ vitogiagulli58@gmail.com )}

Università degli Studi di Bari Aldo Moro Dipartimento Interdisciplinare di Medicina: Universita degli Studi di Bari Aldo Moro Dipartimento Interdisciplinare di Medicina

\section{Research Article}

Keywords: Erectile dysfunction, Charlson comorbidity index, Type 2 diabetes, Prevalence

Posted Date: July 16th, 2021

DOl: https://doi.org/10.21203/rs.3.rs-716137/v1 
License: (c) (i) This work is licensed under a Creative Commons Attribution 4.0 International License. Read Full License 


\section{Abstract}

Introduction. The prevalence of erectile dysfunction (ED) increases along with the burden of chronic diseases. This retrospective study aimed to assess the prevalence and severity of ED according to the levels of chronic comorbidities.

Material and Methods. Two hundred twenty-two outpatients referred to the Outpatients Clinic of Endocrinology and Metabolic Disease of Conversano Hospital (Italy) with ED complaints from January 2018 to December 2019 were retrospectively eligible for this cross-sectional study. The ED severity and comorbidities burden were assessed by the 5 -item International Index of Erectile Function questionnaire (IIEF-5) and Charlson comorbidity index (CCl). A modified index (mCCl) was developed to integrate other common risk factors for ED and was compared to the original tool. The primary outcome was to assess the prevalence of ED according to the severity of $\mathrm{CCl}$. The secondary outcomes included the correlation between 1) IIEF-5 and total testosterone (TT);2) CCI and TT; 3) IIEF-5 and CCI. Finally, the performance of the $\mathrm{CCl}$ and $\mathrm{mCCl}$ were compared.

Results. The overall prevalence of ED increased along with the CCl score: $45 \%$ (5 on 11) for $\mathrm{CCl}=0 ; 95 \%$ (19 on 20) for $\mathrm{CCl}=1 ; 91 \%$ (29 on 32) for $\mathrm{CCl}=2 ; 99 \%$ (158 on 160) for $\mathrm{CCl} \geq 3$ ( $\mathrm{p}<.0001$ ) Moreover, IIEF-5 score was directly correlated with TT levels $(r=0.67 ; p<.0001)$. CCI correlated with both TT levels and IIEF5 score ( $r=-0.34$ and $-0.44 ; p<.0001$, respectively). Finally, a lower IIEF- 5 score was significantly and independently associated with higher age and $\mathrm{CCl}$ as well as lower TT and SHBG. Compared to the CCl, an equal performance was also found with the $\mathrm{mCCl}$.

Discussion. Our results showed that $\mathrm{CCl}$ and $\mathrm{mCCl}$ are reliable tools to assess the presence and severity of ED among outpatients referred to the endocrine center. However, some limitations should be considered, including the number of participants, which appeared underpowered; the single-center experience; possible underestimation of $\mathrm{CCl}$ referred to a diagnostic delay of included comorbidities; arbitrary assignment of burden-points to hypertension dyslipidemia and cigarette smoking.

Conclusion. The present study found that $\mathrm{CCl}$, a validated tool to assess the burden of comorbidities, correlates with both the prevalence and severity of ED. This confirms that ED is a reliable proxy of overall male health, but further studies are needed to confirm this potential application.

\section{Introduction}

Erectile dysfunction (ED) is a sexual disorder characterized by the inability to attain and maintain penis erection sufficient to permit satisfactory sexual intercourse [1]. It constitutes an independent risk factor for poor health, reduced quality of life, and decreased survival. For instance, the presence of ED increases the risk of future cardiovascular events, including myocardial infarction, cerebrovascular events, and allcause mortality $[2,3,4]$. On the other hand, screening men with ED for cardiovascular disease and treating them for both disorders are cost-effective [5]. 
The estimated prevalence of ED increases along with aging. It affects mainly men older than 40 years, with prevalence ranging from $2-9 \%$ in those aged $40-49$ years and from $50-100 \%$ in those older than 70 years [6]. Two main mechanisms have been proposed to explain this phenomenon. First, erectile performance is strictly related to serum total testosterone levels, and an age-related decline in testosterone concentrations is known to occur, as observed in middle-aged and older adults from the European Male Aging Study [7, 8]. Second, the chronic disease burden increases with aging and is independently associated with ED [7, 9]. Lifestyle modifications [10], pharmacotherapy [11], and other interventions such as bariatric surgery [12], targeting these chronic comorbidities, are usually associated with improvements in sexual function.

The Charlson comorbidity index $(\mathrm{CCl})$ is a simple, readily applicable, and validated method to classify chronic comorbidities and predict their impact on death risk. CCI was initially developed for use with medical records data and later adapted for health claims data too. Compared to other comorbidity measures, it considers both the number and severity of 19 pre-defined comorbid conditions, assigning each of them a specific weight $[13,14]$. A PubMed search finds that the original papers have been cited over 9,000 times.

The present cross-sectional study was conceived to investigate the impact of weighted chronic comorbidities on erectile dysfunction prevalence and severity. Notably, we aimed to evaluate the association between the burden of comorbidities assessed by a widely used and validated method (CCl) and the prevalence and severity of ED. In this order, we retrospectively reviewed consecutive adult male patients complaining of ED evaluated at our institution during a given period and re-classified them according to the 5-item International Index of Erectile Function questionnaire (IIEF-5) and the CCI scores by a rigorous study design. Additionally, as common risk factors for ED (e.g., arterial hypertension, dyslipidemia, and smoke) are not included in this tool, we developed a modified index and compared its performance with the original $\mathrm{CCl}$.

\section{Materials And Methods}

\section{Institutional management of patients with erectile dysfunction}

This study was performed in a secondary referral center for endocrine diseases and metabolic disorders accredited by the Italian Society of Andrology and Sexual Medicine (SIAMS), the Outpatients Clinic of Endocrinology, and Metabolic Disease of Conversano Hospital (Italy). Accordingly, patients with sexual and reproductive disorders are referred for the initial and long-term management of male hypogonadism, ED, ejaculation dysfunction, and infertility.

In individuals complaining of ED, the initial evaluation includes the assessment of 1) detailed medical and sexual history, including the evaluation of smoking status; 2) physical examination and anthropometry, including blood pressure, body weight, height, body mass index (BMI), and waist 
circumference (WC); 3) laboratory testing, including fasting plasma glucose, $\mathrm{HbA1c}$, lipid profile, androgens and gonadotropins ( $\mathrm{LH}, \mathrm{FSH}$ ); 4) questionnaires (i.e., IIEF-5). Total testosterone (TT) is assessed in all; in patients with medical conditions affecting the sex hormone-binding globulin (SHBG) levels or whose initial TT concentrations are at or near the lower limit of the normal range (e.g., $300 \mathrm{ng} / \mathrm{dl}$ [10.4 nM/L]), SHBG [14]. The IIEF-5, also known as the Sexual Health Inventory for Men (SHIM), is used to classify the severity of erectile dysfunction [15]. Additional procedures were performed when indicated. Given the high prevalence of cardiovascular disease, a multidisciplinary diagnostic workup is considered. Other laboratory assessments (e.g., PSA, prolactin) and examination (e.g. workup for obstructive sleep apnea) are assessed in subjects with specific signs and associated symptoms. Different procedures (multiple pituitary hormone assessments, genetic analysis, magnetic resonance imaging) are performed in subjects with TT levels $<150 \mathrm{ng} / \mathrm{dl}(5.2 \mathrm{nM} / \mathrm{L})$, given that the risk of an organic form of hypogonadism in this subgroup is high. This approach is based on our previous experience of over more than three decades and is consistent with the most recent literature [16-18].

\section{Study design}

This was a retrospective study. The study period was from January 1, 2018, to December 31, 2019. All consecutive adult male patients complaining of ED, with available data on TT, IIEF-5 questionnaire, and comorbidities, were initially eligible for the study and were searched in the institutional database. Patients were excluded only if the abovementioned data could not be retrieved. All procedures were according to the ethical standards of the University of Bari (protocol number 6454, July 2020) and the Azienda Sanitaria Locale (protocol number: 1294, October 2020) Declaration of Helsinki. To be included in the study, all participants signed the informed consent.

\section{Procedures}

Data collected during the initial evaluation were used. Each patient at the Outpatient Clinic completed the IIEF-5 questionnaire. ED was defined as IIEF-5 score $\leq 21$ and was classified as severe for scores $5-7$, moderate for scores 8-11, mild to moderate for scores 12-16, and mild for 17-21 [16]. Self-reported or newly diagnosed comorbidities were used to calculate the $\mathrm{CCl}$ score. Clinical conditions and associated scores were as follows. Cerebrovascular disease, chronic lung disease, congestive heart failure, connective tissue disease, dementia, diabetes, mild liver disease, myocardial infarction, peripheral vascular disease, and ulcer were assigned 1 point each. Any tumor, diabetes with end-organ damage, hemiplegia, leukemia, lymphoma, and moderate or severe kidney disease (eGFR $<60 \mathrm{ml} / \mathrm{min}$ ) were assigned 2 points. The moderate or severe liver diseases were assigned 3 points each. Acquired immunodeficiency syndrome (AIDS) and solid metastatic tumor were assigned 6 points each [13]. Despite being acknowledged risk factors for ED, arterial hypertension, dyslipidemia, and tobacco smoking had been not initially included in the CCl. Therefore, a modified index (modified Charlson comorbidity index [mCCl]) was developed; the abovementioned disorders were included and assigned 1-point each [4, 18].

\section{Laboratory measurements}


Hormonal parameters were exclusively determined in the laboratory of our hospital as for habitual clinical practice. Each blood specimen was collected between 8, and 9 am after an overnight fast and stored at $-20^{\circ} \mathrm{C}$ until analyzed. Serum LH, FSH, TT, and SHBG were measured by commercial immunometric assays (Immulite, EURO/DPC, UK). Reference range were $7.5 \pm 2.6 \mathrm{IU} / \mathrm{L}$ for $\mathrm{LH}, 6.6 \pm 2.5 \mathrm{IU} / \mathrm{L}$ for $\mathrm{FSH}, 450 \pm 90$ $\mathrm{ng} / \mathrm{dL}$ for TT $(15.6 \pm 3.1 \mathrm{nM} / \mathrm{L})$ and $45.4 \pm 5.1 \mathrm{nM} / \mathrm{L}$ for SHBG. Intra- and inter-assay coefficients of variation of these methods were $<8 \%$ and $<10 \%$, respectively [20].

\section{Statistical analysis}

All analyses were conducted using SAS 9.4 software (SAS Institute, Cary, NC, USA).

The primary outcome was to assess the prevalence of ED according to the level of severity of $\mathrm{CCI}$. The secondary outcomes included the correlation between 1) IIEF-5 and TT;2) CCl and TT;3) IIEF-5 and CCI. Finally, the performance of the $\mathrm{CCl}$ and $\mathrm{mCCl}$ were compared. Continuous variables were expressed as mean and standard deviation (SD) for normally distributed parameters or the median and interquartile range (IQR) in skewed data distribution. Shapiro-Wilk's statistics were used to test normality, and an appropriate function was applied to transform those showing a non-normal distribution. The distribution of patients in each category was described as frequency and proportion. Chi-square test and Chi-squared test for trend were used to verify the association between ED and CCI grouped in four classes (score $=0$, score $=1$, score $=2$, score $\geq 3$ ). Pearson's correlation coefficient or Spearman's non-parametric correlation coefficient was used to testing the secondary outcomes, as necessary, and the Mardia test was used to verify multivariate normality. Univariate and multivariable linear models were applied to evaluate the effect of the parameters age, BMI, TT, LH, FSH, SHBG, albumin, and CCI on the IIEF- 5 scores. The Rsquared value and the residual normality test were used to evaluate the goodness of fit of the multivariable model. All tests of statistical significance were two-tailed, and p-values less than 0.05 were considered statistically significant.

\section{Results}

According to the study protocol, 245 men were initially included. Twenty-two participants were excluded because of missing data related to the IIEF- 5 questionnaire or comorbidities. Therefore, the final study series included 223 men aged 18 to 92 years. One hundred eighty-one patients (81\%) had been diagnosed with type 2 diabetes, 182 (82\%) with arterial hypertension, 192 (86\%) were on statins, and 94 (41\%) were current smokers. None had been treated for erectile dysfunction or other sexual and reproductive disorders. Overall, 211 (95\%) patients were diagnosed with erectile dysfunction: 24 (11\%) with a mild form, $140(66 \%)$ with a mild-to-moderate form, and $47(22 \%)$ with a moderate form.

Table 1. Characteristics of included subjects. 
Subjects included $(n=$ 223)

Age, years \pm sd

Bodyweight, kg [Cl]

Body mass index, $\mathrm{kg} / \mathrm{m}^{2}[\mathrm{Cl}]$

Total testosterone, ng/dL \pm sd

$\mathrm{LH}, \mathrm{IU} / \mathrm{L} \pm \mathrm{sd}$

$\mathrm{FSH}, \mathrm{IU} / \mathrm{L} \pm \mathrm{sd}$

SHBG, nM/L \pm sd

IIEF-5, score [Cl]
$60.8 \pm 11.4$

$89.0[78.3-100.8]$

$31.9[27.0-34.5]$

$313.2 \pm 64.2$

$6.6 \pm 2.5$

$8.3 \pm 5.2$

$38.2 \pm 7.7$

$14[12-16]$

Comorbidities included in the Charlson Comorbidity Index, number of individuals (\%)

1 point

Cerebrovascular disease

$24(10.8 \%)$

Chronic lung disease

$52(23.3 \%)$

Congestive heart failure

$28(12.6 \%)$

Connective tissue disease

$12(5.4 \%)$

Dementia

$5(2.2 \%)$

Diabetes without end-organ damage

$76(34.1 \%)$

Mild liver disease

$110(49.3 \%)$

Myocardial infarction

$46(20.6 \%)$

Peripheral vascular disease

$30(13.5 \%)$

Ulcer

$26(11.7 \%)$

2 points

Any tumor

$35(15.6 \%)$

Diabetes with end-organ damage

105 (47.1\%)

Hemiplegia

$1(0.4 \%)$

Leukemia

$4(1.8 \%)$

Lymphoma

$2(0.9 \%)$

Abbreviations: $\mathbf{s d}$ = standard deviation; $\mathrm{Cl}=$ confidence interval. 


\begin{tabular}{|lll|}
\hline & & \multicolumn{1}{|c|}{ Subjects included $(\mathbf{n}=$} \\
\hline & Moderate or severe kidney disease & $46(20.6 \%)$ \\
\hline 3 points & Moderate or severe liver disease & $8(3.6 \%)$ \\
\hline 6 points & AIDS & $0(0.0 \%)$ \\
\cline { 2 - 4 } & Metastatic solid tumor & $5(2.2 \%)$ \\
\hline Other data, number of individuals (\%) & $182(81.6 \%)$ \\
\hline Arterial hypertension & $192(86.1 \%)$ \\
\hline Dyslipidemia & $94(42.2 \%)$ \\
\hline Current cigarette smokers & \\
\hline Abbreviations: sd = standard deviation; $\mathrm{Cl}=$ confidence interval. & \\
\hline
\end{tabular}

The overall prevalence of ED increased along with the CCl score: $45 \%$ (5 on 11) for $\mathrm{CCl}=0 ; 95 \%$ (19 on 20) for $\mathrm{CCl}=1 ; 91 \%$ (29 on 32) for $\mathrm{CCl}=2 ; 99 \%$ (158 on 160) for $\mathrm{CCl} \geq 3(\mathrm{p}<.0001)$ (Fig. 1). Concerning the secondary outcomes, a direct correlation between the IIEF-5 score and TT levels was found, as expected ( $r=0.67 ; p<.0001)$ (Fig. 2). Additionally, CCl correlated with both TT levels and IIEF-5 score, meaning that a higher burden of weighted comorbidities was associated with lower androgens levels and higher severity of ED ( $r=-0.34$ and $-0.44 ; p<.0001$, respectively) (Figs. 3 and 4$)$. Table 1 shows the results of the univariate and multivariable analyses on parameters associated with the IIEF-5 score. A lower IIEF5 score was significantly and independently associated with higher age and $\mathrm{CCl}$ as well as lower TT and SHBG. The R-squared of the model was 0.65 . The residuals were normally distributed according to Shapiro-Wilk's test, as expected in a goodness of fit.

Table 2. Factors associated with IIEF-5 score. 


\begin{tabular}{|lllll|}
\hline Parameters & Univariate & \multicolumn{3}{c|}{ Multivariable } \\
\cline { 2 - 5 } & Effect $( \pm$ se) & p-value & Effect $( \pm$ se) & p-value \\
\hline Age & $-0.10 \pm 0.02$ & $<.0001$ & $-0.06 \pm 0.02$ & 0.0004 \\
\hline Body mass index & $-0.28 \pm 0.04$ & $<.0001$ & $-0.05 \pm 0.03$ & 0.1115 \\
\hline Total testosterone & $0.04 \pm 0.003$ & $<.0001$ & $0.03 \pm 0.003$ & $<.0001$ \\
\hline LH & $-0.005 \pm 0.06$ & 0.9316 & - & - \\
\hline FSH & $-0.007 \pm 0.05$ & 0.8829 & - & - \\
\hline SHBG & $0.22 \pm 0.03$ & $<.0001$ & $0.10 \pm 0.03$ & 0.0001 \\
\hline Albumina & $-0.05 \pm 0.10$ & 0.6233 & - & - \\
\hline Charlson Comorbidity Index score & $-0.71 \pm 0.10$ & $<.0001$ & $-0.28 \pm 0.08$ & 0.0005 \\
\hline Abbreviations: se = standard error & & & & \\
\hline
\end{tabular}

Since arterial hypertension, dyslipidemia, and cigarette smoking were not included in the original CCl score, we developed a $\mathrm{mCCl}$. As found for the $\mathrm{CCl}$, the overall prevalence of $\mathrm{ED}$ in the four classes of the mCCl differed as follow: $0 \%$ (0 on 1) for $\mathrm{mCCl}=0 ; 57 \%$ (4 on 7) for $\mathrm{mCCl}=1 ; 73 \%$ (8 on 11 ) for $\mathrm{mCCl}=2$; $98 \%$ (199 on 204) for $\mathrm{mCCl} \geq 3$ ( $\mathrm{p}<.0001)$. The correlation between the $\mathrm{mCCl}$, TT, and IIEF- 5 scores was confirmed. Compared to the $\mathrm{CCl}$, an equal performance was found (for details, see supplemental material).

\section{Discussion}

ED is a common sexual disorder in aging males. Since the prevalence of comorbidities increases with age and these are known to be associated with ED, we conducted the present study to evaluate the performance of one of the most validated comorbidities indexes in the risk stratification of ED. A number of 223 consecutive subjects complaining of ED evaluated at our institution were retrospectively enrolled. The IIEF-5 questionnaire assessed the presence and severity of ED. The comorbidities burden was estimated using the standard $\mathrm{CCl}$ and a $\mathrm{mCCl}$, including arterial hypertension, dyslipidemia, and smoke.

The main result of the present study showed that $\mathrm{CCl}$ is a reliable tool to assess the presence and severity of ED. The overall ED prevalence (IIEF $\leq 21)$ increased from $45 \%$ in subjects without comorbidities $(\mathrm{CCl}=$ 0 ) to $99 \%$ in $\mathrm{CCl}$ scores of at least 3 . Additionally, although no subject with a severe form of ED was retrieved, the severity of ED increased along with higher comorbidities burden, reaching moderate forms a prevalence of $28 \%$ in subjects in the latter group. The following correlations supported the reliability of these data. Subjects with lower IIEF-5 were characterized by lower TT, as expected. Lower IIEF-5 and TT 
characterized subjects with higher $\mathrm{CCl}$, as well. Since arterial hypertension, dyslipidemia and smoke were not included in $\mathrm{CCl}$, despite being acknowledged risk factors for $\mathrm{ED}$, we developed a $\mathrm{mCCl}$, including these anamnestic parameters and assigning a weight of " 1 " to each one. The reliability of this modified system was confirmed, although no improved performance was found compared to the standard CCl.

Two practical implications may follow. First, when evaluating a subject complaining of ED, the chance of being affected by ED of any severity is relevant even in those patients with single comorbidity and is close to $100 \%$ in those with isolated moderate or severe liver disease or solid metastatic tumor as well in those with a combination of conditions scoring at least 3. Second, when conducting a population study, the burden of comorbidities assessed by $\mathrm{CCl}$ can be used as a reliable proxy of ED. Several studies evaluating the performance of $\mathrm{CCl}$ in subjects with ED are available in the literature. In 2012, Salonia et al. included 140 men who underwent dynamic penile color-doppler ultrasonography for new-onset ED in the University Vita-Salute San Raffaele [21]. In 2017, Favilla et al. included 425 sexually active men seeking a prostate health screening with table sexual relationship and normal testosterone levels in the Department of Urology of the University of Catania [22]. In 2018, Garcia-Cruz et al. included 430 men referred to seven urological units in Spain [23]. All these studies concluded CCI to be a reliable tool to assess male sexual health. Our study extends current knowledge on this topic based on data from patients referred to an endocrine diseases and metabolic disorders center, and thus exhibiting different clinical characteristics, including an elevated frequency of type 2 diabetes (in our cohort was $81 \%$ ), compared to about $20 \%$ in the cited studies.

The strengths and limitations of the present paper should be discussed. First, a significant number of consecutive men was included in the present study, but this can be considered relatively small compared to the burden of ED in the general population. Second, this was a retrospective cohort study with all procedures consistently performed during the study period by the same medical team. Nevertheless, this resulted in a single-center study. Third, $\mathrm{CCl}$ evaluates the weight of comorbid conditions, and it is well known that a different delay in diagnosis exists for some of them, including diabetes [24] and arterial hypertension [25]; an underestimation of $\mathrm{CCl}$ can be anticipated. We included in the CCl both self-reported and documented diagnoses of chronic conditions during the initial assessment to reduce this burden. Lastly, when the modified CCl was conceived, the authors gave the same weight to the three "novel" variables and arbitrarily established 1 point for each one. Despite the role of hypertension, dyslipidemia, and cigarette smoking in the pathogenesis of ED, the burden of these comorbidities could not be the same and could also be affected by medical treatment and target achievement $[26,27]$.

\section{Conclusion}

The ED prevalence is known to increase with aging, and the same holds for the number of chronic disorders. The present study found that $\mathrm{CCl}$, a validated tool to assess the burden of comorbidities, positively correlates with the prevalence and severity of ED. On the one hand, this confirms that ED is a reliable proxy of overall male health. On the other hand, this implies that a systematic ED assessment among individuals with chronic comorbidities could lead to a diagnosis of this disorder in a significant 
proportion of them, with a higher chance of finding a more severe form among those with a higher burden of comorbidities. From a population study perspective, our data provide the rationale to assess the diagnostic performance of $\mathrm{CCl}$ as a proxy for ED. Further studies are needed to confirm this potential application.

\section{Declarations}

\section{Funding}

This research did not receive any specific grant from any funding agency in the public, commercial, or not-for-profit sector.

\section{Disclosures}

The authors declare that they have no conflict of interest.

\section{Author contributions}

V.A.G. conceived the study. V.A.G., G.L., and V.T. drafted the manuscript. N.B. provided statistical expertise. All the authors read, provided feedback, and approved the final manuscript.

\section{Data availability}

The datasets generated and analyzed during the current study are available from the corresponding author on reasonable request.

\section{References}

1. NIH Consensus Conference. Impotence. NIH Consensus Development Panel on Impotence. JAMA. 1993 July 7;270(1):83-90. PMID: 8510302.

2. Dong JY, Zhang YH, Qin LQ. Erectile dysfunction and risk of cardiovascular disease: meta-analysis of prospective cohort studies. J Am Coll Cardiol. 2011;58(13):1378-85. doi: 10.1016/j.jacc.2011.06.024. PMID: 21920268.

3. Zhao B, Hong Z, Wei Y, Yu D, Xu J, Zhang W. Erectile Dysfunction Predicts Cardiovascular Events as an Independent Risk Factor: A Systematic Review and Meta-Analysis. J Sex Med. 2019;16(7):10051017. doi: 10.1016/j.jsxm.2019.04.004. PMID: 31104857.

4. Burnett AL, Nehra A, Breau RH, Culkin DJ, Faraday MM, Hakim LS, Heidelbaugh J, Khera M, McVary KT, Miner MM, Nelson CJ, Sadeghi-Nejad H, Seftel AD, Shindel AW. Erectile Dysfunction: AUA Guideline. J Urol. 2018;200(3):633-641. doi: 10.1016/j.juro.2018.05.004. PMID: 29746858.

5. Giagulli VA, Moghetti P, Kaufman JM, Guastamacchia E, lacoviello M, Triggiani V. Managing erectile dysfunction in heart failure. Endocr Metab Immune Disord Drug Targets. 2013;13(1):125-34. doi: 10.2174/1871530311313010015. 
6. Shamloul R, Ghanem H. Erectile dysfunction. Lancet. 2013;381(9861):153-65. doi: 10.1016/S01406736(12)60520-0. PMID: 23040455.

7. Wu FC, Tajar A, Pye SR, Silman AJ, Finn JD, O'Neill TW, Bartfai G, Casanueva F, Forti G, Giwercman A, Huhtaniemi IT, Kula K, Punab M, Boonen S, Vanderschueren D; European Male Aging Study Group. Hypothalamic-pituitary-testicular axis disruptions in older men are differentially linked to age and modifiable risk factors: the European Male Aging Study. J Clin Endocrinol Metab. 2008;93(7):273745. doi: 10.1210/jc.2007-1972. PMID: 18270261.

8. Wu FC, Tajar A, Beynon JM, Pye SR, Silman AJ, Finn JD, O'Neill TW, Bartfai G, Casanueva FF, Forti G, Giwercman A, Han TS, Kula K, Lean ME, Pendleton N, Punab M, Boonen S, Vanderschueren D, Labrie F, Huhtaniemi IT; EMAS Group. Identification of late-onset hypogonadism in middle-aged and elderly men. N Engl J Med. 2010;363(2):123-35. doi: 10.1056/NEJMoa0911101. PMID: 20554979.

9. Rastrelli G, Corona G, Maggi M. Both comorbidity burden and low testosterone can explain symptoms and signs of testosterone deficiency in men consulting for sexual dysfunction. Asian $\mathrm{J}$ Androl. 2020;22(3):265-273. doi: 10.4103/aja.aja_61_19. PMID: 31249270.

10. Silva AB, Sousa N, Azevedo LF, Martins C. Physical activity and exercise for erectile dysfunction: systematic review and meta-analysis. Br J Sports Med. 2017;51(19):1419-1424. doi: 10.1136/bjsports-2016-096418. PMID: 27707739.

11. Cai X, Tian Y, Wu T, Cao CX, Bu SY, Wang KJ. The role of statins in erectile dysfunction: a systematic review and meta-analysis. Asian J Androl. 2014;16(3):461-6. doi: 10.4103/1008-682X.123678. PMID: 24556747.

12. Glina FPA, de Freitas Barboza JW, Nunes VM, Glina S, Bernardo WM. What Is the Impact of Bariatric Surgery on Erectile Function? A Systematic Review and Meta-Analysis. Sex Med Rev. 2017;5(3):393402. doi: 10.1016/j.sxmr.2017.03.008. PMID: 28526630.

13. Charlson ME, Pompei P, Ales KL, MacKenzie CR. A new method of classifying prognostic comorbidity in longitudinal studies: development and validation. J Chronic Dis. 1987;40(5):373-83. doi: 10.1016/0021-9681(87)90171-8. PMID: 3558716.

14. Austin SR, Wong YN, Uzzo RG, Beck JR, Egleston BL. Why Summary Comorbidity Measures Such As the Charlson Comorbidity Index and Elixhauser Score Work. Med Care. 2015;53(9):e65-72. doi: 10.1097/MLR.0b013e318297429c. PMID: 23703645.

15. Vermeulen A, Verdonck L, Kaufman JM. A critical evaluation of simple methods for the estimation of free testosterone in serum. J Clin Endocrinol Metab. 1999;84(10):3666-72. doi: 10.1210/jcem.84.10.6079. PMID: 10523012.

16. Rosen RC, Cappelleri JC, Smith MD, Lipsky J, Peña BM. Development and evaluation of an abridged, 5 -item version of the International Index of Erectile Function (IIEF-5) as a diagnostic tool for erectile dysfunction. Int J Impot Res. 1999;11(6):319-26. doi: 10.1038/sj.jij.3900472. PMID: 10637462.

17. Bhasin S, Brito JP, Cunningham GR, Hayes FJ, Hodis HN, Matsumoto AM, Snyder PJ, Swerdloff RS, Wu FC, Yialamas MA. Testosterone Therapy in Men With Hypogonadism: An Endocrine Society 
Clinical Practice Guideline. J Clin Endocrinol Metab. 2018;103(5):1715-1744. doi: 10.1210/jc.201800229. PMID: 29562364.

18. Salonia A, Bettocchi C, Carvalho J, et al. EAU guidelines on Sexual and Reproductive Health. Available at https://uroweb.org/guideline/sexual-and-reproductive-health/.

19. Giagulli VA, Castellana M, Carbone MD, Pelusi C, Ramunni MI, De Pergola G, Guastamacchia E, Triggiani V. Weight loss more than glycemic control may improve testosterone in obese type 2 diabetes mellitus men with hypogonadism. Andrology. 2020;8(3):654-662. doi: 10.1111/andr.12754. PMID: 31919991.

20. Giagulli VA, Carbone MD, Ramunni MI, Licchelli B, De Pergola G, Sabbà C, Guastamacchia E, Triggiani V. Adding liraglutide to lifestyle changes, metformin and testosterone therapy boosts erectile function in diabetic obese men with overt hypogonadism. Andrology. 2015;3(6):1094-103. doi: 10.1111/andr.12099. PMID: 26447645.

21. Salonia A, Castagna G, Saccà A, Ferrari M, Capitanio U, Castiglione F, Rocchini L, Briganti A, Rigatti P, Montorsi F. Is erectile dysfunction a reliable proxy of general male health status? The case for the International Index of Erectile Function-Erectile Function domain. J Sex Med. 2012;9(10):2708-15. doi: 10.1111/j.1743-6109.2012.02869.x. PMID: 22897643.

22. Favilla V, Russo GI, Reale G, Leone S, Castelli T, La Vignera S, Condorelli RA, Calogero AE, Cimino S, Morgia G. Predicting erectile dysfunction in sexually active patients seeking prostate health screening: proposal for a multivariable risk stratification. Int J Impot Res. 2015;27(6):201-5. doi: 10.1038/ijir.2015.15. PMID: 26224573.

23. García-Cruz E, Carrión A, Ajami T, Álvarez M, Correas MÁ, García B, García JV, González C, Portillo JA, Romero-Otero J, Simón C, Torremadé J, Vigués F, Alcaraz A. The Patient's Comorbidity Burden Correlates with the Erectile Dysfunction Severity. Actas Urol Esp. 2018;42(1):57-63. doi: 10.1016/j.acuro.2017.03.011. PMID: 28641871.

24. Harris MI, Klein R, Welborn TA, Knuiman MW. Onset of NIDDM occurs at least 4-7 yr before clinical diagnosis. Diabetes Care. 1992;15(7):815-9. doi: 10.2337/diacare.15.7.815. PMID: 1516497.

25. Imam H, Sundström J, Lind L. Evaluation of time delay between discovery of a high blood pressure in a health screening survey and hypertension diagnosis. Blood Press. 2020;29(6):370-374. doi: 10.1080/08037051.2020.1782726. PMID: 32603237.

26. Mirone V, Imbimbo C, Bortolotti A, Di Cintio E, Colli E, Landoni M, Lavezzari M, Parazzini F. Cigarette smoking as risk factor for erectile dysfunction: results from an Italian epidemiological study. Eur Urol. 2002;41(3):294-7. doi: 10.1016/s0302-2838(02)00005-2. PMID: 12180231.

27. Selvin E, Burnett AL, Platz EA. Prevalence and risk factors for erectile dysfunction in the US. Am J Med. 2007;120(2):151-7. doi: 10.1016/j.amjmed.2006.06.010. PMID: 17275456.

\section{Figures}




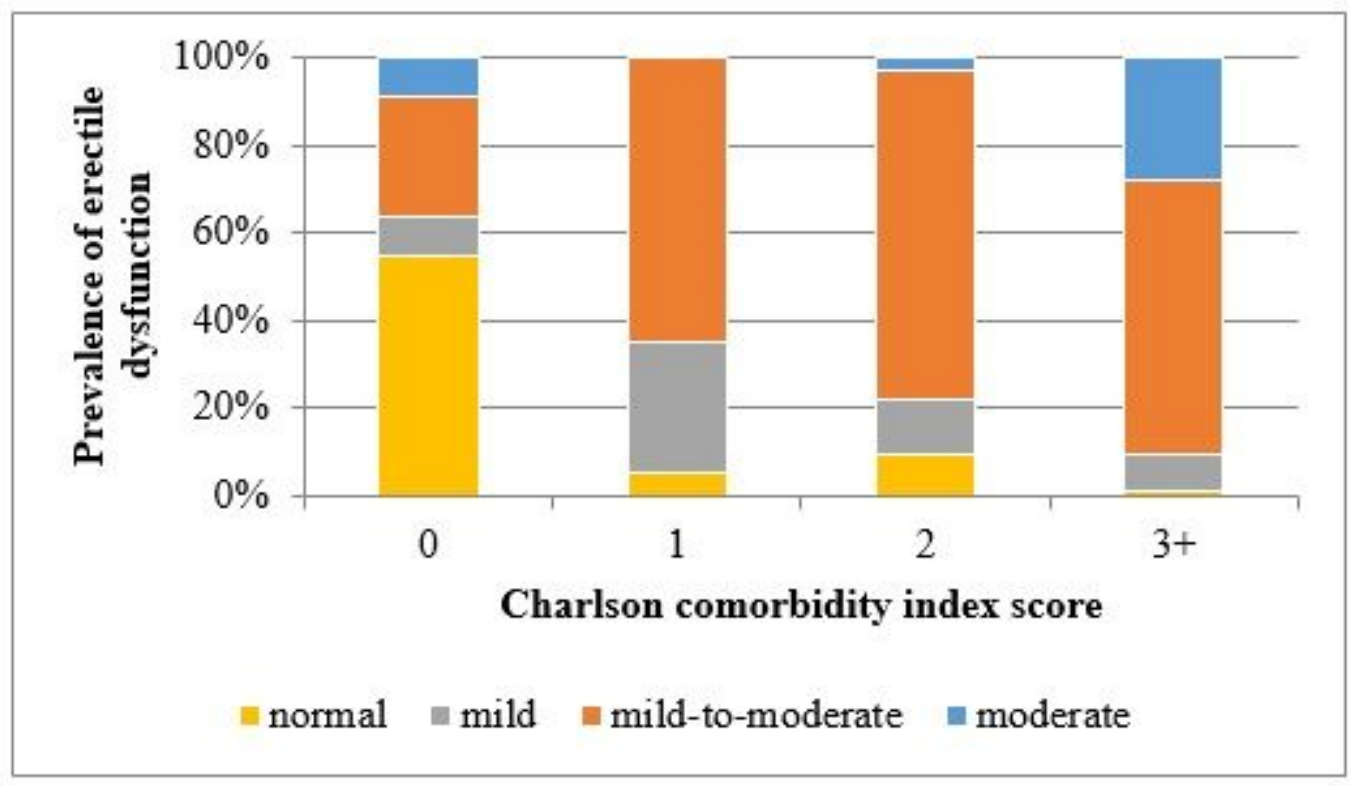

\section{Figure 1}

Prevalence of erectile dysfunction according to the Charlson comorbidity index.

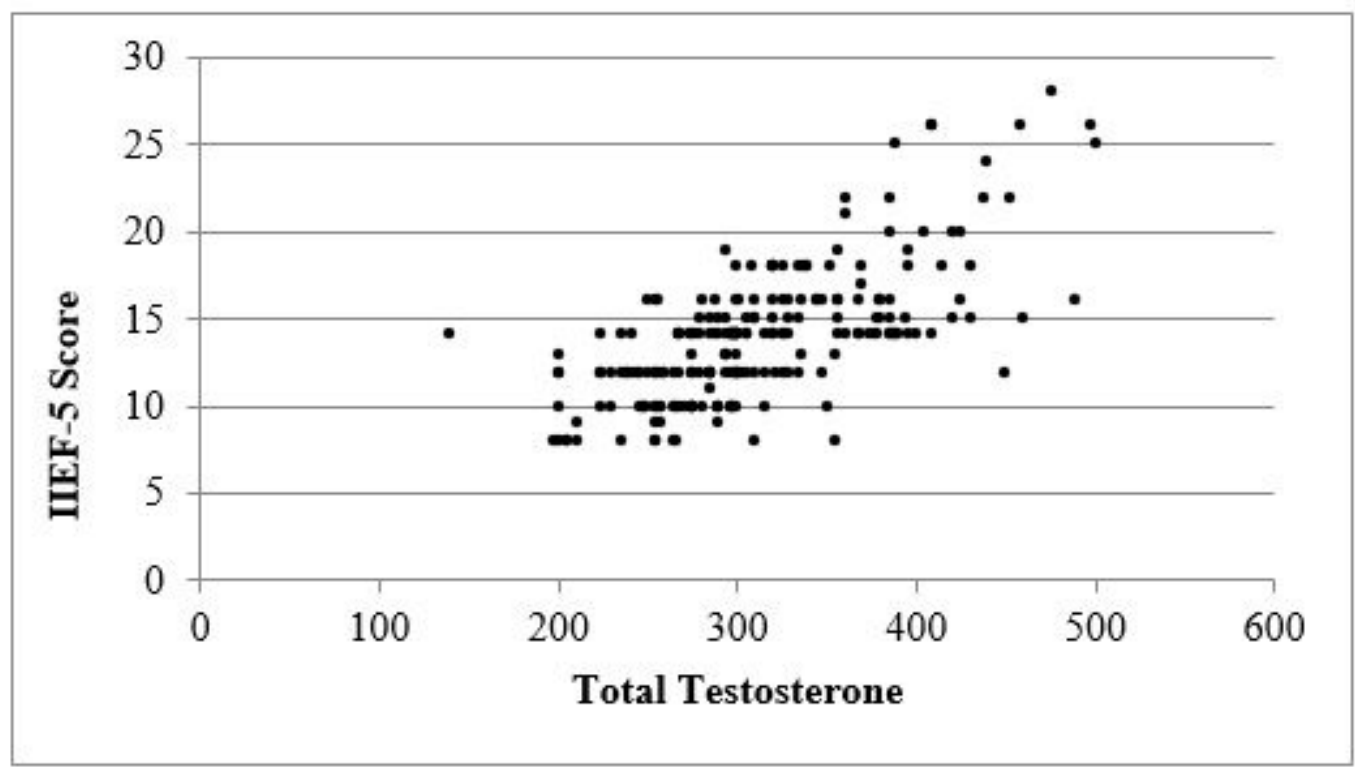

Figure 2

Correlation between IIEF-5 and total testosterone. 


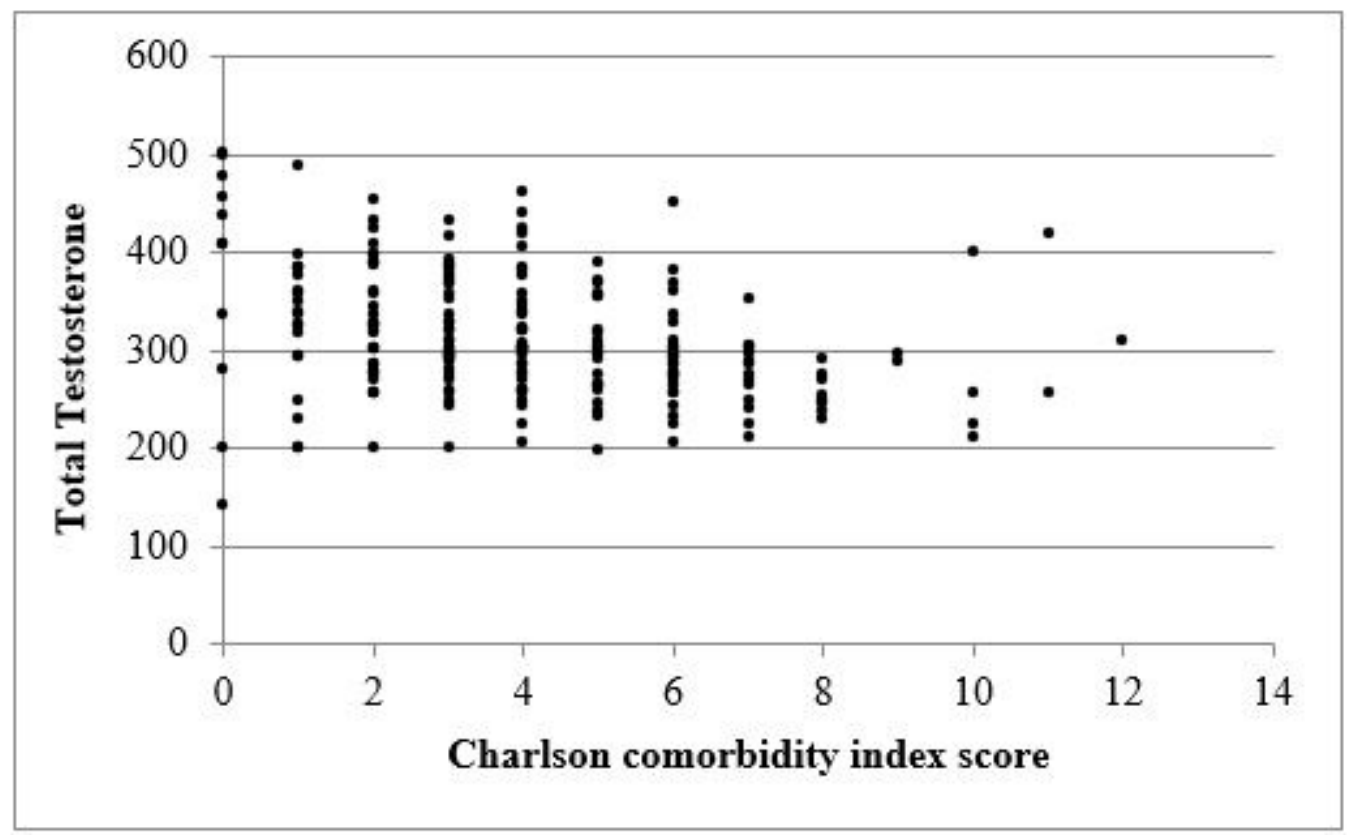

Figure 3

Correlation between Charlson comorbidity index and total testosterone

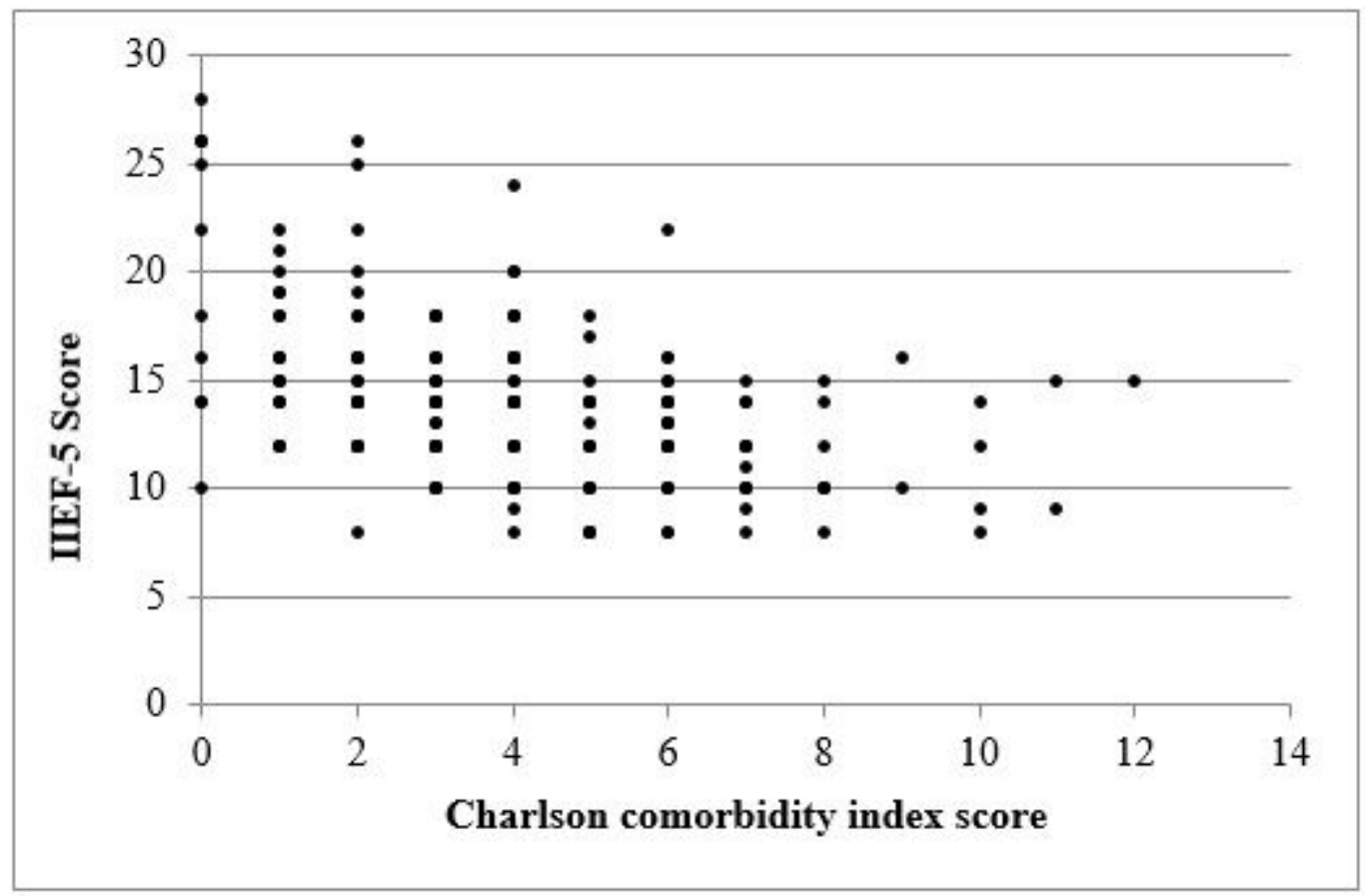

Figure 4

Correlation between Charlson comorbidity index score and IIEF-5 score

\section{Supplementary Files}


This is a list of supplementary files associated with this preprint. Click to download.

- Supplementarymaterial.docx

- FlowDiagramEndocrine.doc

- STROBEchecklistcrosssectional.docx 